\title{
Assessing Cerebrovascular Reactivity in Carotid Steno-Occlusive Disease Using MRI BOLD and ASL Techniques
}

\author{
Renata F. Leoni, ${ }^{1}$ Kelley C. Mazzetto-Betti, ${ }^{1}$ \\ Afonso C. Silva, ${ }^{2}$ Antonio C. dos Santos, ${ }^{1}$ Draulio B. de Araujo, ${ }^{3,4}$ \\ João P. Leite, ${ }^{1}$ and Octavio M. Pontes-Neto ${ }^{1}$ \\ ${ }^{1}$ Department of Neuroscience and Behavioral Sciences, FMRP, University of Sao Paulo, Ribeirao Preto, SP 14049-900, Brazil \\ ${ }^{2}$ Cerebral Microcirculation Unit, Laboratory of Functional and Molecular Imaging, \\ National Institute of Neurological Disorders and Stroke, NIH, Bethesda, MD 20892, USA \\ ${ }^{3}$ Onofre Lopes University Hospital, Federal University of Rio Grande do Norte, Natal, RN 59012-300, Brazil \\ ${ }^{4}$ Brain Institute, Federal University of Rio Grande do Norte, Natal, RN 59020-130, Brazil \\ Correspondence should be addressed to Octavio M. Pontes-Neto, ompneto@usp.br
}

Received 10 February 2012; Revised 17 April 2012; Accepted 17 April 2012

Academic Editor: Kyousuke Kamada

Copyright (c) 2012 Renata F. Leoni et al. This is an open access article distributed under the Creative Commons Attribution License, which permits unrestricted use, distribution, and reproduction in any medium, provided the original work is properly cited.

Impaired cerebrovascular reactivity (CVR), a predictive factor of imminent stroke, has been shown to be associated with carotid steno-occlusive disease. Magnetic resonance imaging (MRI) techniques, such as blood oxygenation level-dependent (BOLD) and arterial spin labeling (ASL), have emerged as promising noninvasive tools to evaluate altered CVR with whole-brain coverage, when combined with a vasoactive stimulus, such as respiratory task or injection of acetazolamide. Under normal cerebrovascular conditions, CVR has been shown to be globally and homogenously distributed between hemispheres, but with differences among cerebral regions. Such differences can be explained by anatomical specificities and different biochemical mechanisms responsible for vascular regulation. In patients with carotid steno-occlusive disease, studies have shown that MRI techniques can detect impaired CVR in brain tissue supplied by the affected artery. Moreover, resulting CVR estimations have been well correlated to those obtained with more established techniques, indicating that BOLD and ASL are robust and reliable methods to assess CVR in patients with cerebrovascular diseases. Therefore, the present paper aims to review recent studies which use BOLD and ASL to evaluate CVR, in healthy individuals and in patients with carotid steno-occlusive disease, providing a source of information regarding the obtained results and the methodological difficulties.

\section{Introduction}

Cerebrovascular reactivity (CVR) is a unique physiologic characteristic of the brain related to the intrinsic ability of encephalic arteries to alter their caliber in response to a vasoactive stimulus [1]. Impaired CVR has been shown to be associated with different pathological conditions, such as hypertension [2-4], arterial stenosis [5-7], and proximal arterial occlusion [8-11]. Severe arterial stenosis or occlusion of proximal vessels of the neck may reduce perfusion pressure in ipsilateral brain regions when collateral flow is insufficient, resulting in autoregulatory vasodilation to maintain regional cerebral blood flow (CBF) within normal limits and then exhausted cerebrovascular reserve capacity, which might contribute to a higher risk of ischemic stroke $[12,13]$. Therefore, investigation of altered CVR may be predictive of imminent stroke in such patients [9, 14-16].

CVR has been generally assessed by positron emission tomography (PET) [17, 18], single-photon emission computed tomography (SPECT) $[19,20]$, and transcranial Doppler (TCD) ultrasonography [21-23]. These methods investigate the residual capacity of cerebral arteries to dilate in response to an increase of carbon dioxide $\left(\mathrm{CO}_{2}\right)$, via $\mathrm{CO}_{2}$ inhalation, breath-holding test $(\mathrm{BHT})$, or acetazolamide (ACZ) administration.

The ability to quantify regional $\mathrm{CBF}$, cerebral blood volume (CBV), and oxygen extraction fraction (OEF) makes PET imaging the gold standard for cerebral perfusion 
evaluation [24]. In healthy subjects, regional differences in CVR have been reported, indicating regional differences in cerebral vascular tone $[25,26]$. Changes in CBF and vasodilatory capacity have been also assessed in patients with cerebral artery occlusion, and results indicated critical hemodynamic status in these patients $[10,27]$. However, although PET has been shown to be useful for CVR assessment, the high cost, technical complexity, limited spatial resolution, and exposure to radiation preclude its routine clinical use.

SPECT, another technique that allows CBF quantification, has also been used to investigate CVR. Studies have shown that reduced regional CVR resulting from arterial occlusion is associated with higher risk of ischemic stroke $[14,15]$. However, this technique requires repeated measurements, before and after ACZ administration, which increases exposure to radiation and introduces errors due to head motion artifacts and heterogeneous washout of tracer [28].

An alternative to either PET or SPECT in the evaluation of CVR is TCD ultrasonography, a simple and noninvasive technique that allows rapid measurements of flow velocities in large cerebral arteries. With a vasodilatory stimulus, it has also provided CVR assessment in healthy subjects and in patients with carotid artery steno-occlusive disease $[1,9$, $16,22,29-31]$. Studies using TCD have also suggested that impaired CVR is predictive of cerebral ischemic events in such patients $[9,32]$. However, lack or poor insonation of the transcranial window may prevent measurements in some subjects [9]. Moreover, despite its high temporal resolution, low cost and reproducibility, TCD's low spatial resolution and, consequently, lack of regional specificity, limits brain mapping.

In the past $10-15$ years, magnetic resonance imaging (MRI) techniques have emerged as promising tools to evaluate cerebral hemodynamics with whole-brain coverage. Blood oxygenation level-dependent (BOLD) images can be acquired with the same vasoactive stimulus cited above, and the percentage of signal change can be used to assess CVR $[33,34]$. Furthermore, the arterial spin labeling (ASL) technique allows CBF measurements at rest and under a vasoactive stimulus, providing quantitative measurements of CVR [4, 35, 36]. In the present paper, a review of several studies which use BOLD and ASL to evaluate CVR, in healthy individuals and in patients with arterial stenoocclusive disease, is presented to provide a useful source of critical information for future improvements on this field.

\section{Vasoactive Challenges to Map CVR}

It has been shown that $\mathrm{CO}_{2}$ has profound effects on cerebrovascular tone, resulting in CBF changes [37-39]. However, arterioles in an injured brain may be less responsive to $\mathrm{CO}_{2}$ alterations, reducing $\mathrm{CBF}$ changes. In patients with occlusive cerebrovascular disease, CVR is generally impaired. Therefore, hypo- and hyper-capnia can be used for CVR mapping in such patients in order to detect brain areas with altered vasodilatory capacity $[5,9,16,26,31,35$, 40]. However, regional differences should be considered when evaluating CVR in patients, making important the investigation of CVR distribution in the brain of healthy individuals.

Hypercapnia can be achieved by BHT or $\mathrm{CO}_{2}$ inhalation, which increase the arterial partial pressure of $\mathrm{CO}_{2}\left(\mathrm{PaCO}_{2}\right)$, without changing the cerebral metabolic rate of oxygen $\left(\mathrm{CMRO}_{2}\right)$. The feasibility and efficacy to investigate CVR with different imaging techniques in combination with hypercapnia have been shown in studies of healthy subjects $[7,22,41]$ and also of patients with carotid stenosis or occlusion $[9,12,16]$. Both challenges, BHT and $\mathrm{CO}_{2}$ inhalation, have provided similar vascular responses [42].

BHT was initially proposed by Ratnatunga and Adiseshiah in 1990 to test cerebral perfusion reserve with TCD [22]. Since then, several studies have used different protocols with BHT to study CVR in healthy subjects and patients. Breath-holding durations from 14 to 30 seconds, after inspiration or expiration, have been reported $[7,8,16,43-$ 47]. The main advantage of BHT is that it can be easily performed during a routine MR examination, with no need of exogenous $\mathrm{CO}_{2}$ source or ACZ injection. However, despite the good practicability, potential shortcomings should be noted. First, the subjects have to be able to understand and perform the task correctly, making it difficult to be used with patients with cognitive deficits or any other problem that preclude BHT execution. Second, BHT leads to different rise rates of $\mathrm{PaCO}_{2}$ in different people, increasing intersubject variability [48]. Moreover, a voluntary act of breath-holding can induce local $\mathrm{CBF}$ and oxygenation changes due to neuronal activation. Recently, the use of cue and feedback mechanisms to control initial inspiration and prolonged breath-holding has been proposed in order to keep the desired $\mathrm{PaCO}_{2}$ level and reduce intersubject variability [49].

On the other hand, $\mathrm{CO}_{2}$ inhalation is a passive task, less dependent on subjects' cooperation. Although it needs an additional apparatus to deliver the gas, it is possible to control the hypercapnia level by the administration of $\mathrm{CO}_{2}$ /air mixture. Fixed $\mathrm{CO}_{2}$ concentrations from 3 to $10 \%$ have been generally used $[9,40-42,50,51]$. Although no adverse effects were observed in such studies, depending on the duration of gas inhalation, $\mathrm{CO}_{2}$ concentrations greater than $7 \%$ were reported to be exhausting [50]. Moreover, $\mathrm{CO}_{2}$ inhalation may be not tolerable to elderly subjects and not safe to patients with obstructive pulmonary diseases [5]. The $\mathrm{CO}_{2}$ inhalation method makes it convenient to deliver the gas, but individual results vary with differences in metabolism amongst subjects. To minimize variability, a method that uses computer-controlled feedback to rapidly set end-tidal oxygen $\left(\mathrm{O}_{2}\right)$ and $\mathrm{CO}_{2}$ concentrations on a breath-by-breath basis to desired target levels was shown to provide a flexible and physiologically well-controlled way to evaluate CVR [52].

Hyperventilation is also a robust and reproducible method to assess CVR. Contrary to $\mathrm{BHT}$ and $\mathrm{CO}_{2}$ inhalation, it is associated with reduction in CBF. The physiological effect of hyperventilation is to reduce $\mathrm{PaCO}_{2}$, increasing $\mathrm{pH}$, which increases cerebrovascular resistance, resulting in marked vasoconstriction and, consequently, reduction in blood flow $[34,37,53]$. A recent respiratory task, named 
"cued deep breathing" (CDB), has been developed to cause transient mild hypocapnia, and consequently vasoconstriction. Resulting high-quality CVR maps comparable to those acquired using BHT indicate CDB as an alternative method in clinical applications [54].

Another vasodilatory substance is the ACZ, a selective inhibitor of carbonic anhydrase that decreases the conversion rate of $\mathrm{CO}_{2}$ to bicarbonate. Injection of ACZ causes $\mathrm{CO}_{2}$ retention in brain tissue, changing extracellular $\mathrm{pH}$, and thus rapidly and markedly increasing $\mathrm{CBF}$, leaving $\mathrm{CMRO}_{2}$ and arterial blood pressure unchanged [20]. Although invasive, ACZ administration has been used to assess CVR in healthy subjects $[1,7]$ and in patients with cerebrovascular disorders $[10,14,15,19]$. In a TCD study, vasodilatory response to ACZ was comparable to the response to BHT and $\mathrm{CO}_{2}$ inhalation [21]. However, another study showed that $\mathrm{CO}_{2}$ inhalation provides a more reproducible increase in blood flow than ACZ [55].

Some studies have investigated the relationship between blood flow and $\mathrm{CO}_{2}$ changes. A linear correlation between BOLD signal changes and the partial pressure of end-tidal $\mathrm{CO}_{2}\left(\mathrm{PetCO}_{2}\right)$ was shown in gray and white matter using a breathing device for controlling $\mathrm{CO}_{2}$ levels [41]. In an ongoing study of our group, the same linear correlation was observed in brain regions supplied by three main cerebral arteries (Figure 1). However, a method using hyperventilation and rebreathing was employed to obtain a wider range of $\mathrm{CO}_{2}$ changes, from hypocapnic to hypercapnic values [29]. Results showed a sigmoidal relationship between CBF and $\mathrm{CO}_{2}$, indicating that CBF may reach a plateau for high levels of $\mathrm{PaCO}_{2}$, as previously reported in human and nonhuman primates $[56,57]$. The linear part of the sigmoidal curve is between $\mathrm{PCO}_{2}$ values of $25-65 \mathrm{mmHg}[41,56]$. Within this range, $\mathrm{CBF}$ normally changes $2-6 \%$ per $\mathrm{mmHg}$ change in $\mathrm{PCO}_{2}[25]$.

\section{CVR Mapping with BOLD}

BOLD signal reflects differences on the magnetic susceptibility of intravascular hemoglobin, depending on whether it is bound to oxygen or not. Oxyhemoglobin is diamagnetic, while deoxyhemoglobin is paramagnetic. An increase in regional CBF surpassing an increase in oxygen consumption results in a reduction of deoxyhemoglobin concentration, increasing local signal intensity in $\mathrm{T} 2 *$-weighted images [58]. Although BOLD images have been mainly used in studies of brain function, they have a great potential to investigate cerebral perfusion and estimate CVR noninvasively, measuring changes in signal intensity after different challenges, such as injection of ACZ [59-61], BHT [5, 42, 45, 51], hyperventilation [34], and $\mathrm{CO}_{2}$ inhalation [40-42, 50, 51, 62, 63].

Under normal cerebrovascular conditions, $\mathrm{CBF}$ in response to vasodilatory stimulus increases globally and homogeneously between hemispheres, but with differences among cerebral regions. Therefore, global increase in BOLD signal has been observed in response to BHT (Figure 2(a)) or $\mathrm{CO}_{2}$ inhalation (Figure 2(b)). Studies using BHT and $\mathrm{CO}_{2}$

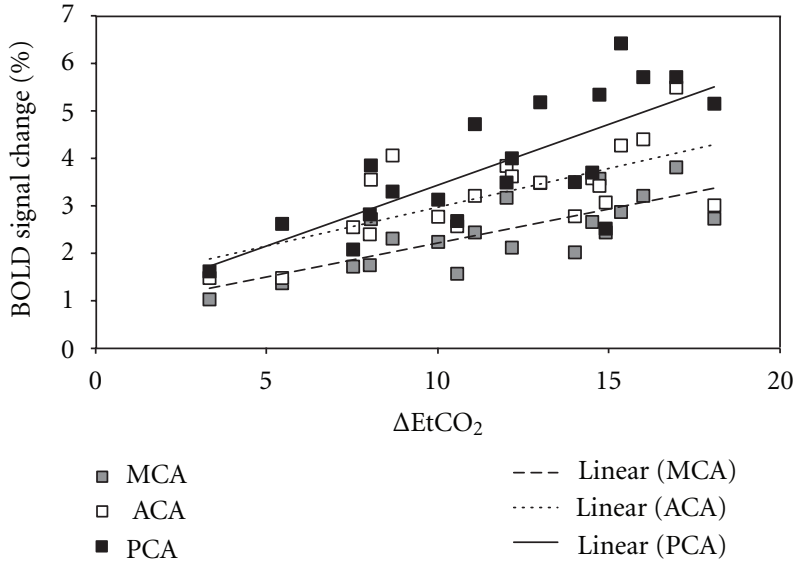

FIGURE 1: Linear correlation between BOLD signal change and $\triangle \mathrm{EtCO}_{2}$ in brain regions supplied by the three main cerebral arteries (anterior: ACA, middle: MCA, and posterior: PCA), for a group of healthy subjects who underwent $\mathrm{CO}_{2}$ inhalation $\left(r^{2}=0.73 \pm 0.04\right)$. Note that the highest increases in BOLD signal were observed in region areas supplies by the posterior cerebral artery (PCA: black squares), and that the lowest BOLD signal amplitudes were observed in areas supplied by the middle cerebral artery (MCA: gray squares).

inhalation have shown significant increase in signal intensity in gray matter, but not in white matter $[33,51,64,65]$. In one of these studies, inhalation of $8 \% \mathrm{CO}_{2}$ increased $\mathrm{PetCO}_{2}$ from $30 \mathrm{mmHg}$ to $46 \mathrm{mmHg}$, resulting in an increase of BOLD signal of $4 \pm 3 \%$ in gray matter and nonsignificant change in white matter [51]. Absence of vasodilation in white matter or insufficient sensitivity of the technique was used to explain that result. However, significant BOLD signal variations in white matter were observed in other studies; although the increase was slower, and the amplitude was lower compared to gray matter $[41,50]$. The same results were previously found in a study using hyperventilation; however, there was a decreasing response due to hypocapnia [34]. In a study using 7\% $\mathrm{CO}_{2}$ inhalation, BOLD signal changes were $5.9 \pm 1.2 \%$ and $1.9 \pm 0.5 \%$, for gray and white matters, respectively [41]. There is evidence that changes in $\mathrm{CBF}$ are mainly due to changes in transit time rather than changes in the number and volume of perfused capillaries [66]. Since the transit time of the hemoglobin is higher in white matter [66], it may explain the differences in BOLD signal changes between gray and white matters.

Regional differences in BOLD responses to a vasoactive stimulus were also observed within gray matter in healthy children and adults [47]. Higher amplitudes were observed in cortical (frontal, occipital, and parieto-occipital cortices) compared to subcortical regions [34, 48]. Within cortical regions, areas supplied by the posterior cerebral artery (PCA), such as the inferior temporal and the occipital gyri, showed the highest BOLD signal amplitudes after BHT or $\mathrm{CO}_{2}$ inhalation $[45,50,67]$. On the other hand, areas supplied by the middle cerebral artery (MCA), such as the middle and inferior frontal gyri, inferior parietal lobes and superior and middle temporal gyri, showed the lowest 


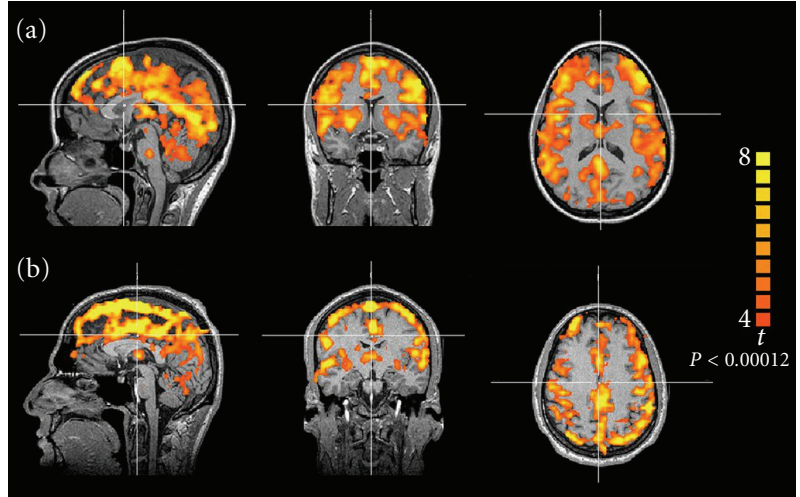

FIgURE 2: Representative BOLD signal maps showing global response to (a) breath-holding test and (b) $\mathrm{CO}_{2}$ inhalation in healthy subjects.

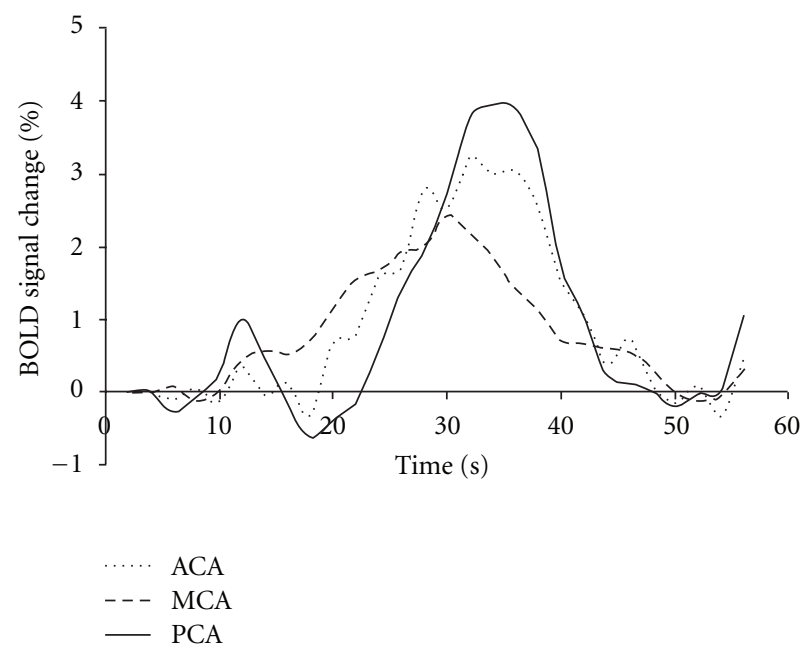

FIgURE 3: BOLD responses to $\mathrm{CO}_{2}$ inhalation in brain regions supplied by the three main cerebral arteries (anterior: ACA, middle: MCA, and posterior: PCA), for healthy subjects.

BOLD signal amplitudes [45]. Different BOLD signal onsets were also observed in different brain regions, indicating an apparent temporal evolution of the response $[43,45,54]$. Figure 3 shows BOLD signal responses to $\mathrm{CO}_{2}$ inhalation in three different brain regions for a group of healthy subjects. Anatomical specificities, such as capillary density, resting $\mathrm{CBV}$, and different biochemical mechanisms responsible for vascular regulation may explain these results $[68,69]$.

Studies using BHT showed that the shape and the amplitude of BOLD responses are not dependent only on brain region, but also on breath-holding duration and technique. Longer breath-holding results in greater BOLD signal amplitude, since greater $\mathrm{CO}_{2}$ levels are presented in blood [43]. Moreover, BOLD responses are dependent on breath-holding technique (Figure 4). Breath-holding after expiration leads to an immediate reduction in arterial partial pressure of $\mathrm{O}_{2}\left(\mathrm{PaO}_{2}\right)$ and increase in $\mathrm{PaCO}_{2}$, resulting in an instantaneous increase in BOLD signal. However, in breathholding after inspiration, $\mathrm{PaO}_{2}, \mathrm{PaCO}_{2}$, arterial $\mathrm{pH}$, and, consequently BOLD signal exhibit a biphasic change. BOLD signal initially decreases during deep inspiration, and then increases due to prolonged breath-holding [43-46].

Several studies assess CVR qualitatively, and the estimation of BOLD signal changes after vasoactive challenges can be just used for interhemispheric comparison within a subject group. To allow reliable comparison between different groups, such as healthy subjects and patients, or between different studies, data should be analyzed quantitatively. Hence, it is interesting the physiological parameter monitoring during MRI exams and further use of $\mathrm{PaCO}_{2}$ or $\mathrm{PetCO}_{2}$ to normalize BOLD responses in the following way:

$$
\mathrm{CVR}_{\mathrm{BOLD}}=100 \times \frac{\mathrm{PSC}_{\text {hyper }}-\mathrm{PSC}_{\text {normo }}}{\mathrm{PetCO}_{2 \text { hyper }}-\mathrm{PetCO}_{2 \text { normo }}},
$$

where $\mathrm{PSC}_{\text {normo }}$ and $\mathrm{PSC}_{\text {hyper }}$ are the percent signal change, and $\mathrm{PetCO}_{2 \text { normo }}$ and PetCO $\mathrm{C}_{2 \text { hyper }}$ are the end-tidal $\mathrm{CO}_{2}$ concentrations during measurements for normocapnia and hypercapnia, respectively.

Kastrup et al. have reported that $5 \%$ of $\mathrm{CO}_{2}$ caused an increase of $13 \pm 2 \mathrm{mmHg}$ in $\mathrm{PetCO}_{2}$ and $2.8 \pm 0.5 \%$ in BOLD response, resulting in an CVR value of $0.21 \pm$ $0.06 \% / \mathrm{mmHg}$ [42]. More recently, Yezhuvath et al. have reported an CVR value of $0.31 \pm 0.08 \% / \mathrm{mmHg}$ in a study with $5 \% \mathrm{CO}_{2}$ inhalation and $\mathrm{PetCO}_{2}$ measurement [70]. Preliminary data from our group showed a similar CVR value $(0.29 \pm 0.07 \% / \mathrm{mmHg})$ for twenty healthy subjects, that were also submitted to a $\mathrm{CO}_{2}$ inhalation protocol.

However, the reliability of CVR quantification with BOLD-MRI in response to hypercapnia can be a concern. Hence, recent studies have investigated the intersubject and interhemispheric variability, and the shortterm reproducibility in groups of healthy subjects. They concluded that CVR quantification have a good betweensession and interhemispheric reproducibility, making the technique useful and safe for CVR assessment in patients with cerebrovascular diseases $[62,63]$.

Another concern about using this technique to assess CVR is that BOLD signal depends on CBF, and also on $\mathrm{CBV}, \mathrm{CMRO}_{2}, \mathrm{PaO}_{2}$, and hematocrit in a complex and not fully understood way. Recent investigations to address this issue showed that BOLD signal response to changes in $\mathrm{PetCO}_{2}$ is directly related to CBF measured with SPECT and ASL, in healthy subjects and in patients with steno-occlusive disease $[7,71]$. Results also showed that BOLD method can differentiate impaired hemispheric CVR from normal CVR.

Furthermore, in patients with unilateral internal carotid artery (ICA) stenosis or occlusion, CVR estimated by BOLDMRI in the MCA territory was found to be well correlated to changes in MCA CBF velocity measured with TCD after inhalation of $3-7 \% \mathrm{CO}_{2}$. In areas of infarction, reduced BOLD $\mathrm{CO}_{2}$ reactivity was observed. In noninfarcted regions ipsilateral to the stenosis or occlusion, CVR was impaired $[11,40]$. In such areas, TCD was not able to estimate CVR, showing an advantage of BOLD-MRI technique.

To prevent ischemic stroke, patients with severe carotid stenosis often benefit from interventions, such as carotid endarterectomy (CEA) and carotid angioplasty with stent 


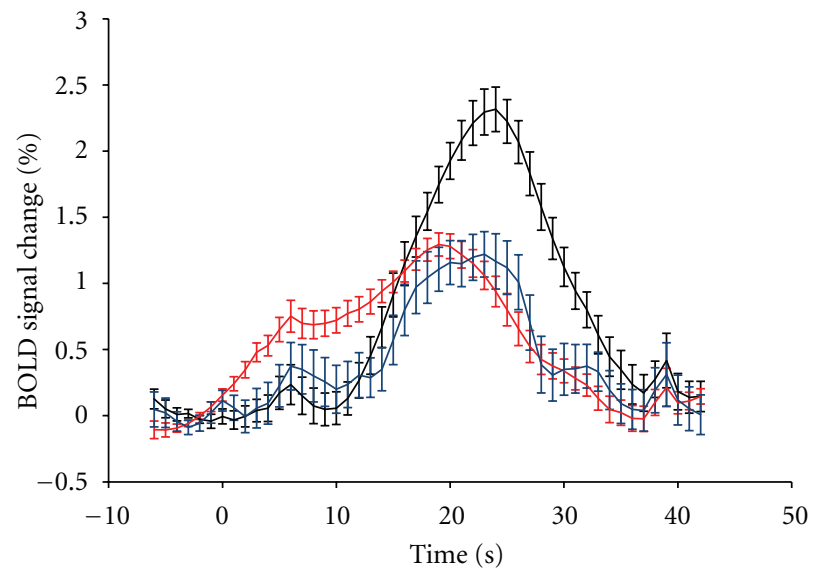

(a)

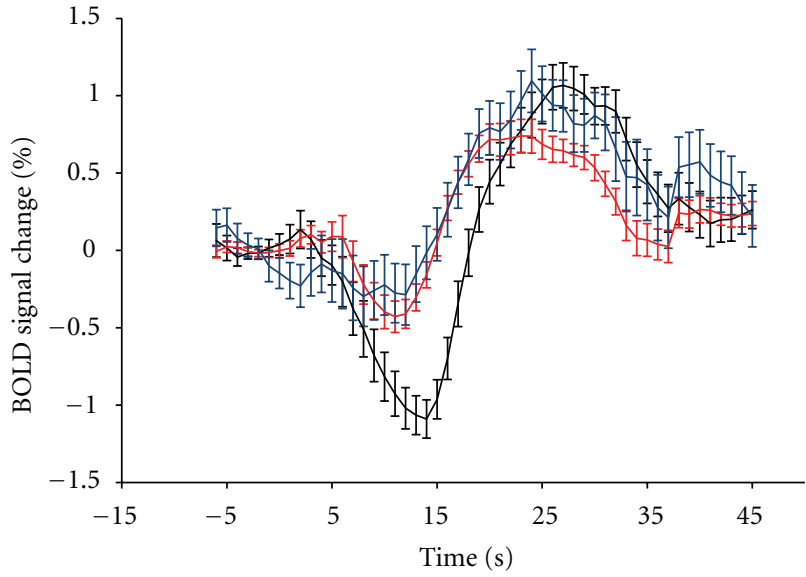

(b)

FIGURE 4: Average BOLD response to breath-holding of 15 seconds after (a) expiration and (b) inspiration on brain regions supplied by the three main cerebral arteries (anterior: blue, middle: red, and posterior: black), for a group of healthy subjects. Reproduced from Leoni et al. [45].

placement (CAS) [72-74]. Recently, studies have reported that patients with carotid stenosis, who showed impaired CVR before carotid intervention, had great CVR improvement after CEA or CAS $[5,6]$. This finding is probably due to an improvement in vasodilatory ability, indicating return of vascular reserve capacity. Although such patients may benefit from greater hemodynamic improvements, hyperperfusion is still a concern that can lead to hemorrhage [75]. In fact, a condition called cerebral hyperperfusion syndrome (CHS) after carotid intervention has been classically described as an acute neurologic deficit occurring from hours to days following a carotid procedure and represents a spectrum of clinical symptoms ranging from severe unilateral headache, to seizures and focal neurologic defects, to intracerebral hemorrhage in its most severe form. The mechanism appears to be related to increased regional cerebral blood flow secondary to loss of cerebrovascular autoregulation [76, 77].

It is worth noting that the above observations about the influence of $\mathrm{CO}_{2}$ in BOLD response and the impaired CVR in cerebrovascular diseases have important impact on fMRI brain activation studies. Since perfusion is not homogeneous throughout human brain, the statistical maps obtained in typical fMRI studies may reflect, partially, perfusional differences. Therefore, it has been recently proposed the use of hypercapnia, mainly BHT, as a way to normalize fMRI results, which would take into account individual perfusion responses [78-80]. However, the discussion of these calibration methods is not in the scope of this review.

\section{CVR Mapping with ASL}

Quantitative maps of CBF can be obtained with ASL, a noninvasive MRI technique which uses water protons presented in arterial blood as an endogenous perfusion tracer. This technique is based on the differentiation between stationary spins and spins flowing with blood, which is labeled using radiofrequency pulses. As labeled intravascular spins reach the capillaries and do exchanges with brain tissue through blood-brain barrier, tissue magnetization is altered, making it possible to obtain images in which contrast is proportional to cerebral perfusion [81-83]. There are different ASL methods, but all of them are based on the same principle. Images are acquired distally to the labeling plane after labeled blood flowed to the target tissue. Perfusion map is then obtained by subtracting labeled and control images. The latter are acquired without spin labeling.

Therefore, perfusion images with high spatial resolution showing quantitative CBF values in $\mathrm{mL} / \mathrm{g} / \mathrm{min}$ can be calculated [83], allowing detection of global and regional altered perfusion and data comparison in longitudinal studies [84]. Despite its advantages, ASL has limited signal-to-noise ratio and complex flow kinetics, requiring measurements of $\mathrm{T} 1$ values for blood and tissue, labeling efficiency and arterial transit time for absolute CBF quantification [85]. However, its noninvasiveness is very suitable for perfusion studies in patients with renal insufficiency, for repetitive follow-up studies and for pediatric investigations in which exogenous contrasts or radioactive tracers are not recommended.

Furthermore, ASL can be used to assess CVR, by mapping CBF under normo- and hyper-capnic conditions [36]. CVR can be calculated as $\% / \mathrm{mmHg}$ in the following way:

$$
\mathrm{CVR}_{\mathrm{ASL}}=\frac{100}{\mathrm{CBF}_{\text {normo }}} \times \frac{\mathrm{CBF}_{\text {hyper }}-\mathrm{CBF}_{\text {normo }}}{\text { PetCO }_{2 \text { hyper }}-\text { PetCO }_{2 \text { normo }}},
$$

where $\mathrm{CBF}_{\text {normo }}$ and $\mathrm{CBF}_{\text {hyper }}$ are the cerebral blood flow, and $\mathrm{PetCO}_{2 \text { normo }}$ and $\mathrm{PetCO}_{2 \text { hyper }}$ are the end-tidal $\mathrm{CO}_{2}$ concentrations during measurements for normocapnia and hypercapnia, respectively.

In healthy subjects, global CBF increase was observed in response to hypercapnia [36]. Yen et al. have shown satisfactory CVR measurement reproducibility between session days and good sensitivity to small changes, which is important when investigating disease or treatment [86]. Noth et al. have 
compared perfusion MRI measurements at $1.5 \mathrm{~T}$ and $3.0 \mathrm{~T}$, and observed no CBF and CVR differences between magnetic fields. CVR values in healthy subjects were $4.3 \pm 0.7 \% / \mathrm{mmHg}$ and $4.5 \pm 1.3 \% / \mathrm{mmHg}$ for $1.5 \mathrm{~T}$ and $3.0 \mathrm{~T}$, respectively [36]. Other two recent studies showed regional difference in CVR values in healthy subjects. CVR in areas irrigated by ICA was lower than in areas irrigated by the basilar artery $[87,88]$.

ASL perfusion imaging has been clinically applied in the evaluation of cerebrovascular diseases [89]. Recent studies have demonstrated altered hemodynamics and regional CBF in patients with arterial stenosis [90]. As cited in previous session, Mandell et al. have found significant correlation between CVR values measured with BOLD and ASL in patients. For example, in a 57-year-old male patient with left ICA occlusion, CVR values in gray matter of the ipsilateral hemisphere were $\left(\mathrm{CVR}_{\mathrm{BOLD}}=0.22 \% / \mathrm{mmHg}\right.$; $\mathrm{CVR}_{\mathrm{ASL}}=1.79 \% / \mathrm{mmHg}$ ) significantly lower than the values of the contralateral hemisphere $\left(\mathrm{CVR}_{\mathrm{BOLD}}=0.33 \% / \mathrm{mmHg}\right.$; $\left.\mathrm{CVR}_{\mathrm{ASL}}=3.35 \% / \mathrm{mmHg}\right)[71]$.

More recently, Bokkers et al. performed two studies to assess CVR with ASL and ACZ administration. One study was in patients with carotid artery stenosis, and results showed decreased CVR in brain tissue supplied by the symptomatic ICA, when compared to regions supplied by the contralateral ICA and to CVR results in control subjects [88]. The other study was performed in patients that presented ICA occlusion. Again, results showed impaired CVR in brain tissue on the side of the occluded ICA [35]. Moreover, regional $\mathrm{CBF}$ and $\mathrm{CVR}$ values measured with ASL were significantly correlated with those measured with SPECT [91]. Therefore, ASL in combination with vascular challenge is able to evaluate CVR in patients with ICA stenosis or occlusion.

\section{Conclusions}

Patients with arterial stenosis or occlusion have a high risk for ischemic stroke, which is significantly influenced by the degree of CVR impairment and the collateral flow development. Therefore, several studies have been investigating CVR to identify patients with increased risk and to better plan the treatment. For these purposes, MRI technique may play an important role as a noninvasive tool capable to assess cerebral reserve capacity in combination with a vascular challenge.

Assessment of CVR with BOLD-MRI is an alternative to PET, SPECT, and TCD and can be easily included in clinical examinations. It has the advantage of mapping whole brain with good spatial resolution, allowing investigation of CVR regional distribution. And despite providing semiquantitative data, studies showed that the method is robust, reliable, and reproducible, allowing assessment of CVR in patients with cerebrovascular diseases. On the other hand, ASL is a quantitative method to assess whole-brain CBF and CVR. Moreover, it has been used to selectively label blood flowing through specific cerebral arteries, which allows investigation of collateral flow. Recently, CBF-ASL has been used in many clinical applications, such as in arterial stenoocclusive disease, but also in hypertension [92], diabetes [93], Alzheimer's disease [94], epilepsy [95], and brain tumor
[96], in which resting perfusion is believed to be altered. However, in some cases, regional CBF is normal, but cerebral reserve capacity may be exhausted. In such cases, CVR mapping may be a powerful tool to investigate compromised hemodynamics.

In addition to the known disadvantages of each method, other factors may interfere with the results of arterial stenosis or occlusion investigation, such as the small number of subjects in some studies [5-7, 35, 40, 90]; inclusion of both symptomatic and asymptomatic patients; inclusion of both arterial stenosis and occlusion in the same study; no investigation of other factors that alter CVR, such as age [97], gender [98], hypertension [92], and diabetes [93].

In conclusion, functional MRI techniques (BOLD and ASL) are an alternative to PET, SPECT, and TCD for the assessment of CVR and can be easily included during an MR imaging scan for clinical purposes. Those methods are robust, reliable and reproducible, allowing assessment of CVR in patients with cerebrovascular diseases. Therefore, the clinical impact of a combination of those MRI techniques as a tool to assess CVR in patients with carotid stenoocclusive disease and to select the best recanalization strategy for patients with carotid stenosis requires further clinical investigation.

\section{Acknowledgments}

This research was supported by FAPESP (2011/15874-0) and partially by the Intramural Research Program of the NIH, NINDS (Alan P. Koretsky, scientific director).

\section{References}

[1] N. Schwertfeger, P. Neu, P. Schlattmann, H. Lemke, I. Heuser, and M. Bajbouj, "Cerebrovascular reactivity over time course in healthy subjects," Journal of the Neurological Sciences, vol. 249, no. 2, pp. 135-139, 2006.

[2] C. Iadecola and R. L. Davisson, "Hypertension and cerebrovascular dysfunction," Cell Metabolism, vol. 7, no. 6, pp. 476-484, 2008.

[3] C. Iadecola, L. Park, and C. Capone, "Threats to the mind: aging, amyloid, and hypertension," Stroke, vol. 40, supplement 3, pp. S40-S44, 2009.

[4] R. F. Leoni, F. F. Paiva, E. C. Henning et al., "Magnetic resonance imaging quantification of regional cerebral blood flow and cerebrovascular reactivity to carbon dioxide in normotensive and hypertensive rats," NeuroImage, vol. 58, no. 1, pp. 75-81, 2011.

[5] T. Y. Chang, H. L. Liu, T. H. Lee et al., "Change in cerebral perfusion after carotid angioplasty with stenting is related to cerebral vasoreactivity: a study using dynamic susceptibilityweighted contrast-enhanced MR imaging and functional MR imaging with a breath-holding paradigm," American Journal of Neuroradiology, vol. 30, no. 7, pp. 1330-1336, 2009.

[6] S. D. Goode, N. Altaf, D. P. Auer, and S. T. MacSweeney, "Carotid endarterectomy improves cerebrovascular reserve capacity preferentially in patients with preoperative impairment as indicated by asymmetric BOLD response to hypercapnia," European Journal of Vascular and Endovascular Surgery, vol. 38, no. 5, pp. 546-551, 2009. 
[7] A. Shiino, Y. Morita, A. Tsuji et al., "Estimation of cerebral perfusion reserve by blood oxygenation level-dependent imaging: comparison with single-photon emission computed tomography," Journal of Cerebral Blood Flow \& Metabolism, vol. 23, no. 1, pp. 121-135, 2003.

[8] A. Apruzzese, M. Silvestrini, R. Floris et al., "Cerebral hemodynamics in asymptomatic patients with internal carotid artery occlusion: a dynamic susceptibility contrast MR and transcranial Doppler study," American Journal of Neuroradiology, vol. 22, no. 6, pp. 1062-1067, 2001.

[9] H. Markus and M. Cullinane, "Severely impaired cerebrovascular reactivity predicts stroke and TIA risk in patients with carotid artery stenosis and occlusion," Brain, vol. 124, no. 3, pp. 457-467, 2001.

[10] H. Okazawa, H. Yamauchi, H. Toyoda, K. Sugimoto, Y. Fujibayashi, and Y. Yonekura, "Relationship between vasodilatation and cerebral blood flow increase in impaired hemodynamics: a PET study with the acetazolamide test in cerebrovascular disease," Journal of Nuclear Medicine, vol. 44, no. 12, pp. 1875-1883, 2003.

[11] S. Ziyeh, J. Rick, M. Reinhard, A. Hetzel, I. Mader, and O. Speck, "Blood oxygen level-dependent MRI of cerebral $\mathrm{CO}_{2}$ reactivity in severe carotid stenosis and occlusion," Stroke, vol. 36, no. 4, pp. 751-756, 2005.

[12] M. Silvestrini, E. Troisi, M. Matteis, L. M. Cupini, and C. Caltagirone, "Transcranial Doppler assessment of cerebrovascular reactivity in symptomatic and asymptomatic severe carotid stenosis," Stroke, vol. 27, no. 11, pp. 1970-1973, 1996.

[13] M. W. Webster, M. S. Makaroun, D. L. Steed, H. A. Smith, D. W. Johnson, and H. Yonas, "Compromised cerebral blood flow reactivity is a predictor of stroke in patients with symptomatic carotid artery occlusive disease," Journal of Vascular Surgery, vol. 21, no. 2, pp. 338-344, 1995, discussion 344-345.

[14] S. Kuroda, K. Houkin, H. Kamiyama, K. Mitsumori, Y. Iwasaki, and H. Abe, "Long-term prognosis of medically treated patients with internal carotid or middle cerebral artery occlusion: can acetazolamide test predict it?" Stroke, vol. 32, no. 9, pp. 2110-2116, 2001.

[15] K. Ogasawara, A. Ogawa, and T. Yoshimoto, "Cerebrovascular reactivity to acetazolamide and outcome in patients with symptomatic internal carotid or middle cerebral artery occlusion: a xenon-133 single-photon emission computed tomography study," Stroke, vol. 33, no. 7, pp. 1857-1862, 2002.

[16] F. Vernieri, P. Pasqualetti, M. Matteis et al., "Effect of collateral blood flow and cerebral vasomotor reactivity on the outcome of carotid artery occlusion," Stroke, vol. 32, no. 7, pp. 15521558, 2001.

[17] J. M. Gibbs, R. J. Wise, K. L. Leenders, and T. Jones, "Evaluation of cerebral perfusion reserve in patients with carotid-artery occlusion," The Lancet, vol. 1, no. 8372, pp. 310314, 1984.

[18] T. Nezu, C. Yokota, T. Uehara et al., "Preserved acetazolamide reactivity in lacunar patients with severe white-matter lesions: ${ }^{15} \mathrm{O}$-labeled gas and $\mathrm{H}_{2} \mathrm{O}$ positron emission tomography studies," Journal of Cerebral Blood Flow \& Metabolism, vol. 32, pp. 844-850, 2012.

[19] K. Aso, K. Ogasawara, M. Sasaki et al., "Preoperative cerebrovascular reactivity to acetazolamide measured by brain perfusion SPECT predicts development of cerebral ischemic lesions caused by microemboli during carotid endarterectomy," European Journal of Nuclear Medicine and Molecular Imaging, vol. 36, no. 2, pp. 294-301, 2009.

[20] S. Vorstrup, L. Henriksen, and O. B. Paulson, "Effect of acetazolamide on cerebral blood flow and cerebral metabolic rate for oxygen," Journal of Clinical Investigation, vol. 74, no. 5, pp. 1634-1639, 1984.

[21] M. Muller, M. Voges, U. Piepgras, and K. Schimrigk, "Assessment of cerebral vasomotor reactivity by transcranial Doppler ultrasound and breath-holding: a comparison with acetazolamide as vasodilatory stimulus," Stroke, vol. 26, no. 1, pp. 96-100, 1995.

[22] C. Ratnatunga and M. Adiseshiah, "Increase in middle cerebral artery velocity on breath holding: a simplified test of cerebral perfusion reserve," European Journal of Vascular Surgery, vol. 4, no. 5, pp. 519-523, 1990.

[23] C. K. Willie, F. L. Colino, D. M. Bailey et al., "Utility of transcranial Doppler ultrasound for the integrative assessment of cerebrovascular function," Journal of Neuroscience Methods, vol. 196, no. 2, pp. 221-237, 2011.

[24] T. J. Carroll, V. Teneggi, M. Jobin et al., "Absolute quantification of cerebral blood flow with magnetic resonance, reproducibility of the method, and comparison with $\mathrm{H}_{2}^{15} \mathrm{O}$ positron emission tomography," Journal of Cerebral Blood Flow \& Metabolism, vol. 22, no. 9, pp. 1149-1156, 2002.

[25] H. Ito, I. Kanno, and H. Fukuda, "Human cerebral circulation: positron emission tomography studies," Annals of Nuclear Medicine, vol. 19, no. 2, pp. 65-74, 2005.

[26] H. Ito, I. Yokoyama, H. Iida et al., "Regional differences in cerebral vascular response to $\mathrm{P}_{a} \mathrm{CO}_{2}$ changes in humans measured by positron emission tomography," Journal of Cerebral Blood Flow \& Metabolism, vol. 20, no. 8, pp. 12641270, 2000.

[27] E. M. Nemoto, H. Yonas, R. R. Pindzola et al., "PET OEF reactivity for hemodynamic compromise in occlusive vascular disease," Journal of Neuroimaging, vol. 17, no. 1, pp. 54-60, 2007.

[28] J. L. Moretti, M. Caglar, and P. Weinmann, "Cerebral perfusion imaging tracers for SPECT: which one to choose?" Journal of Nuclear Medicine, vol. 36, no. 3, pp. 359-363, 1995.

[29] J. A. Claassen, R. Zhang, Q. Fu, S. Witkowski, and B. D. Levine, "Transcranial Doppler estimation of cerebral blood flow and cerebrovascular conductance during modified rebreathing," Journal of Applied Physiology, vol. 102, no. 3, pp. 870-877, 2007.

[30] M. Muller and K. Schimrigk, "Vasomotor reactivity and pattern of collateral blood flow in severe occlusive carotid artery disease," Stroke, vol. 27, no. 2, pp. 296-299, 1996.

[31] L. Soinne, J. Helenius, T. Tatlisumak et al., "Cerebral hemodynamics in asymptomatic and symptomatic patients with highgrade carotid stenosis undergoing carotid endarterectomy," Stroke, vol. 34, no. 7, pp. 1655-1661, 2003.

[32] L. L. Yeo and V. K. Sharma, "Role of transcranial doppler ultrasonography in cerebrovascular disease," Recent Patents on CNS Drug Discovery, vol. 5, no. 1, pp. 1-13, 2010.

[33] A. Kastrup, T. Q. Li, A. Takahashi, G. H. Glover, and M. E. Moseley, "Functional magnetic resonance imaging of regional cerebral blood oxygenation changes during breath holding," Stroke, vol. 29, no. 12, pp. 2641-2645, 1998.

[34] S. Posse, U. Olthoff, M. Weckesser, L. Jancke, H. W. MullerGartner, and S. R. Dager, "Regional dynamic signal changes during controlled hyperventilation assessed with blood oxygen level-dependent functional MR imaging," American Journal of Neuroradiology, vol. 18, no. 9, pp. 1763-1770, 1997.

[35] R. P. Bokkers, M. J. van Osch, C. J. Klijn, L. J. Kappelle, and J. Hendrikse, "Cerebrovascular reactivity within perfusion territories in patients with an internal carotid artery occlusion," Journal of Neurology, Neurosurgery and Psychiatry, vol. 82, no. 9, pp. 1011-1016, 2011. 
[36] U. Noth, G. E. Meadows, F. Kotajima, R. Deichmann, D. R. Corfield, and R. Turner, "Cerebral vascular response to hypercapnia: determination with perfusion MRI at 1.5 and 3.0 Tesla using a pulsed arterial spin labeling technique," Journal of Magnetic Resonance Imaging, vol. 24, no. 6, pp. 1229-1235, 2006.

[37] R. D. Conn and J. S. Cole, "The cardiac apex impulse. Clinical and angiographic correlations," Annals of Internal Medicine, vol. 75, no. 2, pp. 185-191, 1971.

[38] C. F. Schmidt and S. S. Kety, "Recent studies of cerebral blood flow and cerebral metabolism in man," Transactions of the Association of American Physicians, vol. 60, no. 1, pp. 52-58, 1947.

[39] P. Novack, H. A. Shenkin, L. Bortin, B. Goluboff, and A. M. Soffe, "The effects of carbon dioxide inhalation upon the cerebral blood flow and cerebral oxygen consumption in vascular disease," The Journal of Clinical Investigation, vol. 32, no. 8, pp. 696-702, 1953.

[40] D. J. Lythgoe, S. C. Williams, M. Cullinane, and H. S. Markus, "Mapping of cerebrovascular reactivity using bold magnetic resonance imaging," Magnetic Resonance Imaging, vol. 17, no. 4, pp. 495-502, 1999.

[41] F. H. van der Zande, P. A. Hofman, and W. H. Backes, "Mapping hypercapnia-induced cerebrovascular reactivity using BOLD MRI," Neuroradiology, vol. 47, no. 2, pp. 114-120, 2005.

[42] A. Kastrup, G. Kruger, T. Neumann-Haefelin, and M. E. Moseley, "Assessment of cerebrovascular reactivity with functional magnetic resonance imaging: comparison of $\mathrm{CO}_{2}$ and breath holding," Magnetic Resonance Imaging, vol. 19, no. 1, pp. 1320, 2001.

[43] K. C. Andrade, O. M. Pontes-Neto, J. P. Leite, A. C. Santos, O. Baffa, and D. B. de Araujo, "Quantitative aspects of brain perfusion dynamic induced by BOLD fMRI," Arquivos de Neuro-Psiquiatria, vol. 64, no. 4, pp. 895-898, 2006.

[44] A. Kastrup, J. Dichgans, M. Niemeier, and M. Schabet, "Changes of cerebrovascular $\mathrm{CO}_{2}$ reactivity during normal aging," Stroke, vol. 29, no. 7, pp. 1311-1314, 1998.

[45] R. F. Leoni, K. C. Mazzeto-Betti, K. C. Andrade, and D. B. de Araujo, "Quantitative evaluation of hemodynamic response after hypercapnia among different brain territories by fMRI," NeuroImage, vol. 41, no. 4, pp. 1192-1198, 2008.

[46] T. Q. Li, A. Kastrup, A. M. Takahashi, and M. E. Moseley, "Functional MRI of human brain during breath holding by BOLD and FAIR techniques," NeuroImage, vol. 9, no. 2, pp. 243-249, 1999.

[47] M. E. Thomason, B. E. Burrows, J. D. Gabrieli, and G. H. Glover, "Breath holding reveals differences in fMRI BOLD signal in children and adults," NeuroImage, vol. 25, no. 3, pp. 824-837, 2005.

[48] A. Kastrup, G. Kruger, G. H. Glover, T. Neumann-Haefelin, and M. E. Moseley, "Regional variability of cerebral blood oxygenation response to hypercapnia," NeuroImage, vol. 10, no. 6, pp. 675-681, 1999.

[49] M. E. Thomason and G. H. Glover, "Controlled inspiration depth reduces variance in breath-holding-induced BOLD signal," NeuroImage, vol. 39, no. 1, pp. 206-214, 2008.

[50] E. Rostrup, I. Law, M. Blinkenberg et al., "Regional differences in the CBF and BOLD responses to hypercapnia: a combined PET and fMRI study," NeuroImage, vol. 11, no. 2, pp. 87-97, 2000.

[51] A. Vesely, H. Sasano, G. Volgyesi et al., "MRI mapping of cerebrovascular reactivity using square wave changes in endtidal $\mathrm{PCO}_{2}$," Magnetic Resonance in Medicine, vol. 45, no. 6, pp. 1011-1013, 2001.
[52] R. G. Wise, K. T. Pattinson, D. P. Bulte et al., "Dynamic forcing of end-tidal carbon dioxide and oxygen applied to functional magnetic resonance imaging," Journal of Cerebral Blood Flow \& Metabolism, vol. 27, no. 8, pp. 1521-1532, 2007.

[53] S. Naganawa, D. G. Norris, S. Zysset, and T. Mildner, "Regional differences of fMR signal changes induced by hyperventilation: comparison between SE-EPI and GE-EPI at 3-T," Journal of Magnetic Resonance Imaging, vol. 15, no. 1, pp. 23-30, 2002.

[54] M. G. Bright, D. P. Bulte, P. Jezzard, and J. H. Duyn, "Characterization of regional heterogeneity in cerebrovascular reactivity dynamics using novel hypocapnia task and BOLD fMRI," NeuroImage, vol. 48, no. 1, pp. 166-175, 2009.

[55] K. Kazumata, N. Tanaka, T. Ishikawa, S. Kuroda, K. Houkin, and K. Mitsumori, "Dissociation of vasoreactivity to acetazolamide and hypercapnia: comparative study in patients with chronic occlusive major cerebral artery disease," Stroke, vol. 27, no. 11, pp. 2052-2058, 1996.

[56] J. A. Madden, "The effect of carbon dioxide on cerebral arteries," Pharmacology and Therapeutics, vol. 59, no. 2, pp. 229-250, 1993.

[57] M. Reivich, "Arterial $\mathrm{Pco}_{2}$ and cerebral hemodynamics," The American Journal of Physiology, vol. 206, pp. 25-35, 1964.

[58] S. Ogawa, T. M. Lee, A. R. Kay, and D. W. Tank, "Brain magnetic resonance imaging with contrast dependent on blood oxygenation," Proceedings of the National Academy of Sciences of the United States of America, vol. 87, no. 24, pp. 9868-9872, 1990.

[59] H. Bruhn, A. Kleinschmidt, H. Boecker, K. D. Merboldt, W. Hanicke, and J. Frahm, "The effect of acetazolamide on regional cerebral blood oxygenation at rest and under stimulation as assessed by MRI," Journal of Cerebral Blood Flow \& Metabolism, vol. 14, no. 5, pp. 742-748, 1994.

[60] P. Hedera, S. Lai, J. S. Lewin et al., "Assessment of cerebral blood flow reserve using functional magnetic resonance imaging," Journal of Magnetic Resonance Imaging, vol. 6, no. 5, pp. 718-725, 1996.

[61] B. Mukherjee, M. Preece, G. C. Houston et al., "Mapping of the cerebral response to acetazolamide using graded asymmetric spin echo EPI," Magnetic Resonance Imaging, vol. 23, no. 9, pp. 907-920, 2005.

[62] S. D. Goode, S. Krishan, C. Alexakis, R. Mahajan, and D. P. Auer, "Precision of cerebrovascular reactivity assessment with use of different quantification methods for hypercapnia functional MR imaging," American Journal of Neuroradiology, vol. 30, no. 5, pp. 972-977, 2009.

[63] A. Kassner, J. D. Winter, J. Poublanc, D. J. Mikulis, and A. P. Crawley, "Blood-oxygen level dependent MRI measures of cerebrovascular reactivity using a controlled respiratory challenge: reproducibility and gender differences," Journal of Magnetic Resonance Imaging, vol. 31, no. 2, pp. 298-304, 2010.

[64] E. Rostrup, H. B. Larsson, P. B. Toft et al., "Functional MRI of $\mathrm{CO}_{2}$ induced increase in cerebral perfusion," $N M R$ in Biomedicine, vol. 7, no. 1-2, pp. 29-34, 1994.

[65] A. E. Stillman, X. Hu, and M. Jerosch-Herold, "Functional MRI of brain during breath holding at 4 T," Magnetic Resonance Imaging, vol. 13, no. 6, pp. 893-897, 1995.

[66] D. Bereczki, L. Wei, T. Otsuka et al., "Hypercapnia slightly raises blood volume and sizably elevates flow velocity in brain microvessels," American Journal of Physiology, vol. 264, no. 5, pp. H1360-H1369, 1993.

[67] R. G. Wise, K. Ide, M. J. Poulin, and I. Tracey, "Resting fluctuations in arterial carbon dioxide induce significant low frequency variations in BOLD signal," NeuroImage, vol. 21, no. 4, pp. 1652-1664, 2004. 
[68] P. A. Bandettini and E. C. Wong, "A hypercapnia-based normalization method for improved spatial localization of human brain activation with fMRI," NMR in Biomedicine, vol. 10, no. 4-5, pp. 197-203, 1997.

[69] T. L. Davis, K. K. Kwong, R. M. Weisskoff, and B. R. Rosen, "Calibrated functional MRI: mapping the dynamics of oxidative metabolism," Proceedings of the National Academy of Sciences of the United States of America, vol. 95, no. 4, pp. 18341839, 1998.

[70] U. S. Yezhuvath, K. Lewis-Amezcua, R. Varghese, G. Xiao, and $\mathrm{H}$. Lu, "On the assessment of cerebrovascular reactivity using hypercapnia BOLD MRI," NMR in Biomedicine, vol. 22, no. 7, pp. 779-786, 2009.

[71] D. M. Mandell, J. S. Han, J. Poublanc et al., "Mapping cerebrovascular reactivity using blood oxygen level-dependent MRI in patients with arterial steno-occlusive disease: comparison with arterial spin labeling MRI," Stroke, vol. 39, no. 7, pp. 2021-2028, 2008.

[72] C. Warlow, B. Farrell, A. Fraser, P. Sandercock, and J. Slattery, "Randomised trial of endarterectomy for recently symptomatic carotid stenosis: final results of the MRC European Carotid Surgery Trial (ECST)," The Lancet, vol. 351, no. 9113, pp. 1379-1387, 1998.

[73] G. G. Ferguson, M. Eliasziw, H. W. K. Barr et al., "The North American Symptomatic Carotid Endarterectomy Trial: surgical results in 1415 patients," Stroke, vol. 30, no. 9, pp. 1751-1758, 1999.

[74] R. L. Sacco, R. Adams, G. Albers et al., "Guidelines for prevention of stroke in patients with ischemic stroke or transient ischemic attack: a statement for healthcare professionals from the American Heart Association/American Stroke Association council on stroke-Co-sponsored by the council on cardiovascular radiology and intervention. The American Academy of Neurology affirms the value of this guideline," Stroke, vol. 37, no. 2, pp. 577-617, 2006.

[75] G. S. Sfyroeras, C. D. Karkos, G. Arsos et al., "Cerebral hyperperfusion after carotid stenting: a transcranial Doppler and SPECT study," Vascular and Endovascular Surgery, vol. 43, no. 2, pp. 150-156, 2009.

[76] K. G. Moulakakis, S. N. Mylonas, G. S. Sfyroeras, and V. Andrikopoulos, "Hyperperfusion syndrome after carotid revascularization," Journal of Vascular Surgery, vol. 49, no. 4, pp. 1060-1068, 2009.

[77] W. N. van Mook, R. J. Rennenberg, G. W. Schurink et al., "Cerebral hyperperfusion syndrome," The Lancet Neurology, vol. 4 , no. 12, pp. 877-888, 2005.

[78] D. F. Abbott, H. I. Opdam, R. S. Briellmann, and G. D. Jackson, "Brief breath holding may confound functional magnetic resonance imaging studies," Human Brain Mapping, vol. 24, no. 4, pp. 284-290, 2005.

[79] D. A. Handwerker, A. Gazzaley, B. A. Inglis, and M. D'Esposito, "Reducing vascular variability of fMRI data across aging populations using a breathholding task," Human Brain Mapping, vol. 28, no. 9, pp. 846-859, 2007.

[80] M. E. Thomason, L. C. Foland, and G. H. Glover, "Calibration of BOLD fMRI using breath holding reduces group variance during a cognitive task," Human Brain Mapping, vol. 28, no. 1, pp. 59-68, 2007.

[81] E. L. Barbier, L. Lamalle, and M. Decorps, "Methodology of brain perfusion imaging," Journal of Magnetic Resonance Imaging, vol. 13, no. 4, pp. 496-520, 2001.

[82] F. Calamante, D. L. Thomas, G. S. Pell, J. Wiersma, and R. Turner, "Measuring cerebral blood flow using magnetic resonance imaging techniques," Journal of Cerebral Blood Flow \& Metabolism, vol. 19, no. 7, pp. 701-735, 1999.

[83] J. A. Detre, J. Wang, Z. Wang, and H. Rao, "Arterial spinlabeled perfusion MRI in basic and clinical neuroscience," Current Opinion in Neurology, vol. 22, no. 4, pp. 348-355, 2009.

[84] S. Petcharunpaisan, J. Ramalho, and M. Castillo, "Arterial spin labeling in neuroimaging," World Journal of Radiology, vol. 2, no. 10, pp. 384-398, 2010.

[85] X. Golay, E. T. Petersen, I. Zimine, and T. C. Lim, "Arterial spin labeling: a one-stop-shop for measurement of brain perfusion in the clinical settings," in Proceedings of the 29th Annual International Conference of IEEE Engineering in Medicine and Biology Society (EMBC '07), pp. 4320-4323, Lyon, France, August 2007.

[86] Y. F. Yen, A. S. Field, E. M. Martin et al., "Test-retest reproducibility of quantitative CBF measurements using FAIR perfusion MRI and acetazolamide challenge," Magnetic Resonance in Medicine, vol. 47, no. 5, pp. 921-928, 2002.

[87] R. P. Bokkers, J. P. Bremmer, B. N. van Berckel et al., "Arterial spin labeling perfusion MRI at multiple delay times: a correlative study with $\mathrm{H}_{2}^{15} \mathrm{O}$ positron emission tomography in patients with symptomatic carotid artery occlusion," Journal of Cerebral Blood Flow \& Metabolism, vol. 30, no. 1, pp. 222-229, 2010.

[88] R. P. Bokkers, M. J. van Osch, H. B. van der Worp, G. J. de Borst, W. P. Mali, and J. Hendrikse, "Symptomatic carotid artery stenosis: impairment of cerebral autoregulation measured at the brain tissue level with arterial spin-labeling MR imaging," Radiology, vol. 256, no. 1, pp. 201-208, 2010.

[89] J. A. Detre and D. C. Alsop, "Perfusion magnetic resonance imaging with continuous arterial spin labeling: methods and clinical applications in the central nervous system," European Journal of Radiology, vol. 30, no. 2, pp. 115-124, 1999.

[90] A. C. Roc, J. Wang, B. M. Ances, D. S. Liebeskind, S. E. Kasner, and J. A. Detre, "Altered hemodynamics and regional cerebral blood flow in patients with hemodynamically significant stenoses," Stroke, vol. 37, no. 2, pp. 382-387, 2006.

[91] Y. Uchihashi, K. Hosoda, I. Zimine et al., "Clinical application of arterial spin-labeling MR imaging in patients with carotid stenosis: quantitative comparative study with single-photon emission CT," American Journal of Neuroradiology, vol. 32, no. 8, pp. 1545-1551, 2011.

[92] I. Hajjar, P. Zhao, D. Alsop, and V. Novak, "Hypertension and cerebral vasoreactivity: a continuous arterial spin labeling magnetic resonance imaging study," Hypertension, vol. 56, no. 5, pp. 859-864, 2010.

[93] D. Last, D. C. Alsop, A. M. Abduljalil et al., "Global and regional effects of type 2 diabetes on brain tissue volumes and cerebral vasoreactivity," Diabetes Care, vol. 30, no. 5, pp. 11931199, 2007.

[94] S. Cantin, M. Villien, O. Moreaud et al., "Impaired cerebral vasoreactivity to $\mathrm{CO}_{2}$ in Alzheimer's disease using BOLD fMRI," Neurolmage, vol. 58, no. 2, pp. 579-587, 2011.

[95] R. L. Wolf, D. C. Alsop, I. Levy-Reis et al., "Detection of mesial temporal lobe hypoperfusion in patients with temporal lobe epilepsy by use of arterial spin labeled perfusion MR imaging," American Journal of Neuroradiology, vol. 22, no. 7, pp. 13341341, 2001.

[96] D. Zaca, J. Hua, and J. J. Pillai, "Cerebrovascular reactivity mapping for brain tumor presurgical planning," World Journal of Clinical Oncology, vol. 2, no. 7, pp. 289-298, 2011. 
[97] A. Riecker, W. Grodd, U. Klose et al., "Relation between regional functional MRI activation and vascular reactivity to carbon dioxide during normal aging," Journal of Cerebral Blood Flow \& Metabolism, vol. 23, no. 5, pp. 565-573, 2003.

[98] M. Matteis, E. Troisi, B. C. Monaldo, C. Caltagirone, and M. Silvestrini, "Age and sex differences in cerebral hemodynamics: a transcranial Doppler study," Stroke, vol. 29, no. 5, pp. 963967, 1998. 


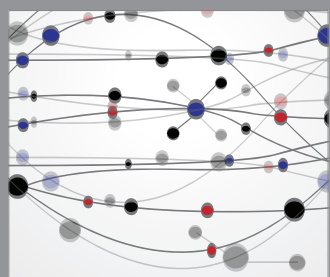

The Scientific World Journal
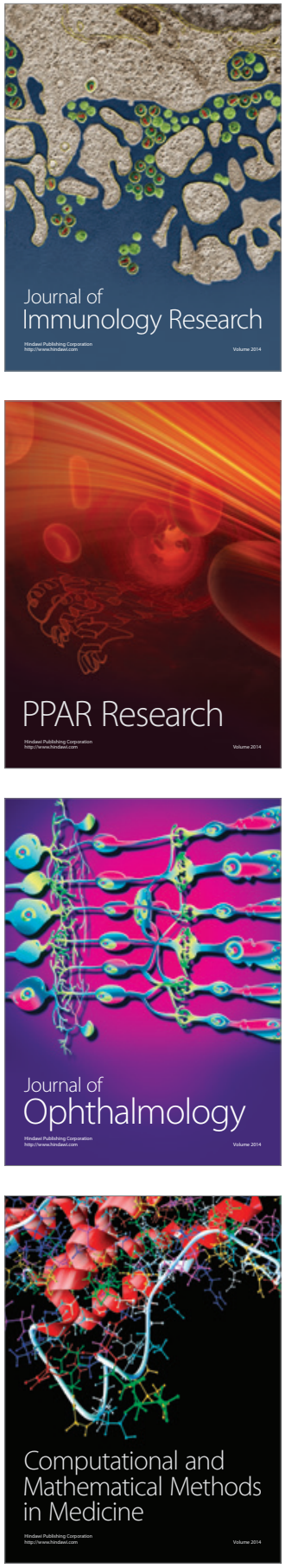

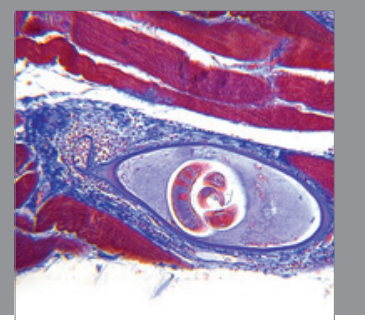

Gastroenterology

Research and Practice
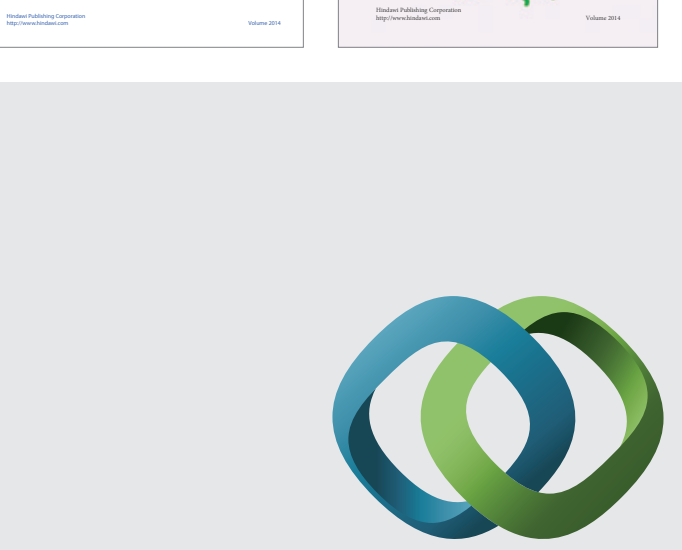

\section{Hindawi}

Submit your manuscripts at

http://www.hindawi.com
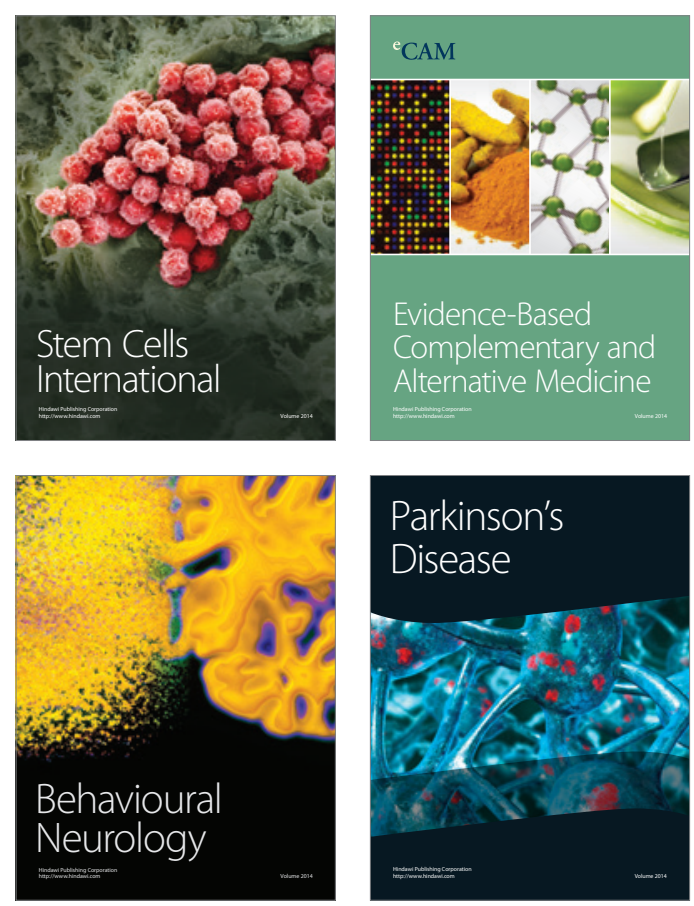

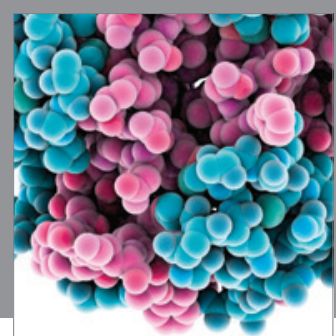

Journal of
Diabetes Research

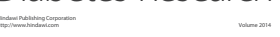

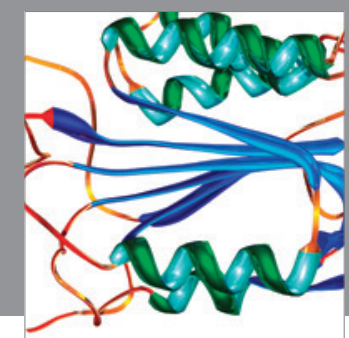

Disease Markers
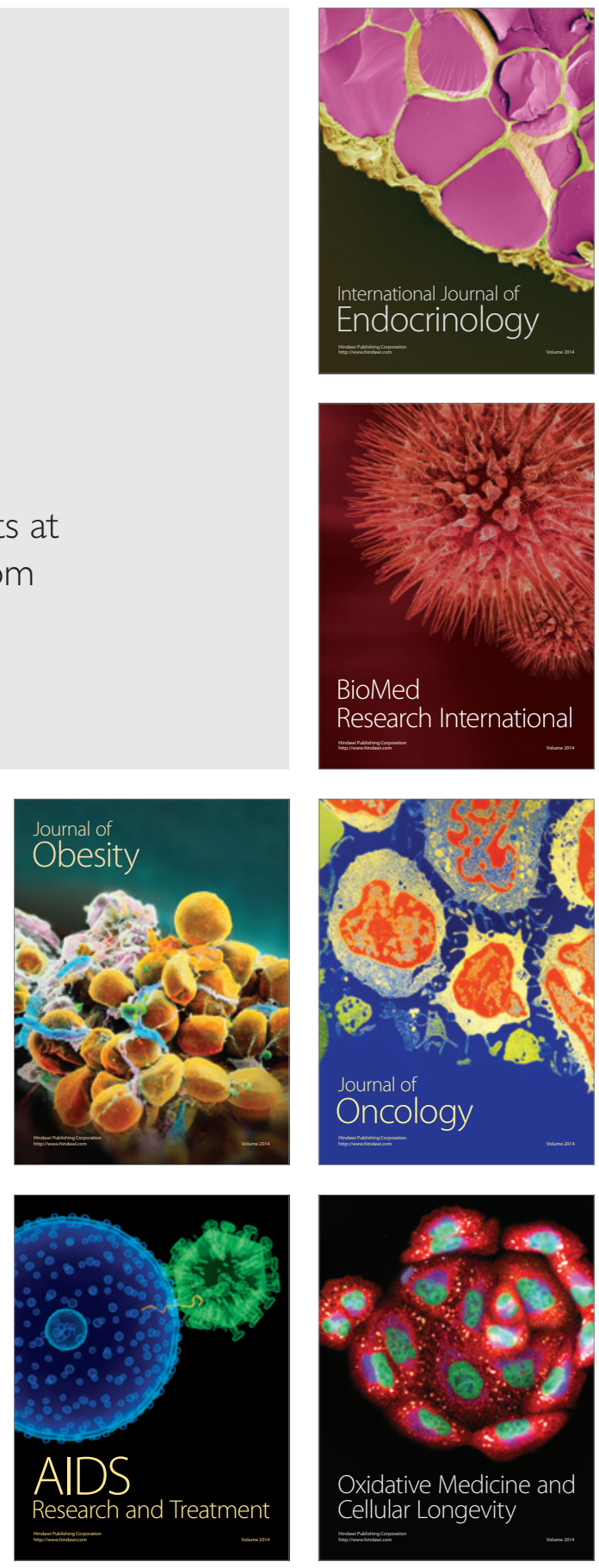